\title{
Hair: A Review of Prominent Scalp Hair Changes throughout a Females' Life
}

\section{Dalia Gamal Aly*}

Department of Dermatology and Venereology, National Research Center, El-Bhoos St., Dokky, Giza, Egypt

\begin{abstract}
Throughout a females' life, scalp hair growth is variable in its extent and time course. Age-related changes can occur to it including changes in the hair cycling, hair density, hair diameter and pigmentation, and possibly in structural qualities of the hair fiber. Evidence is starting to appear that environmental factors also contribute, possibly in a major way, to the changes that we observe in scalp hair growth as women get older. These changes are most pronounced in female pattern hair loss. Determining the cause or causes of hair loss in women can be difficult and should be guided by the patient's history, including, the pattern of hair loss, other medical conditions, the use of hair treatments, and the family history as well as by the physical examination to differentiate between female pattern hair loss (FPHL) and telogen effluvium (TE). Topical anti-aging compounds include photoprotectors and antioxidants. In the absence of another way to reverse hair graying, hair colorants remain the mainstay of recovering lost hair color. Topical liposome targeting for melanins, genes, and proteins selectively to hair follicles are currently under investigation.
\end{abstract}

Keywords: Androgenetic alopecia (AGA); Aging; Hair; Telogen effluvium

\section{Introduction}

The appearance of hair plays an important role in people's overall physical appearance and self-perception. Nowadays, the desire to look youthful plays a bigger role than the past [1]. As in all other tissues in the human body, the hair follicle is susceptible to the ravages of age. In contrast to other tissues, age-related changes in hair growth vary across the skin, both in their extent and in their time course. For example, the growth of terminal hairs in the axilla and pubic region begins around puberty, is maximal by mid-twenties and then gradually declines. Beard growth starts and peaks later but is then maintained throughout life, and may increase in women in old age. This review concentrates on female scalp hair growth, where the effect of age, is variable and difficult to understand. Changes can occur in hair cycling, hair density, hair diameter and pigmentation, and possibly in structural qualities of the hair fiber. Evidence is starting to appear that environmental factors also contribute, possibly in a major way, to the changes observed in scalp hair growth as women get older [2].

Hair loss, is also a very common presenting symptom in females, and more than one third of women have clinically significant hair loss during their lifetime. It seems likely, although unproven, that genes are involved especially when onset occurs early [1]. What is the pathology and what we can expect as the result of a natural ageing process is difficult to define. But in evaluating the patient complaining of hair loss, while true pathology must always be considered, the clinician needs to be aware of how age affects hair growth [2]. These changes form the focus of this review.

\section{The Hair Cycle}

Scalp hair grows in cycles, with each hair follicle undergoing 10 to 30 cycles in its lifetime. Normally, each hair follicle cycles independently, so that while some hairs are growing, others are resting and others are shedding. Thus, the density of the scalp hair and the total number of scalp hairs remain stable [3].

The period of active hair growth is known as anagen which is heralded by the onset of mitotic activity in epithelial cells overlying the dermal papilla at the base of the follicle [4]. The duration of this phase is responsible for determining the final length of the hair. Anagen may continue for several years and can last about 2 to 8 years, so that very long hairs are produced. Under normal circumstances, $80-90 \%$ of hair follicles on the human scalp are in anagen at one time. These hairs are anchored deeply into the subcutaneous fat and cannot be pulled out easily [2].

At the end of anagen, epithelial cell division declines and ceases and the hair follicles prepare themselves for the resting phase. This transition period of a hair follicle from growth to rest is called catagen (involutionary phase) which lasts 4-6 weeks. During this phase, the lower part of the follicle involutes by programmed apoptosis leading to a collapse to the hair follicle [3].

The base of the follicle, together with its dermal papilla, moves upwards, and eventually regresses. The period between the completion of follicular regression and the onset of the next anagen phase is termed telogen and lasts 2 to 3 months. As compared with anagen hair, telogen hair is located higher in the skin and can be pulled out relatively easily [2].

At the end of the telogen phase, older hairs that have finished their life will fall out or shed and this is termed exogen. Hair follicles may remain in a state of latency, also known as kenogen, for a prolonged period after the club hair is shed before a new cycle begins [5].

Courtois et al., [6] conducted a longitudinal study on adult men for over 14 years and showed that the mean duration of anagen in scalp hair follicles declined with advancing age together with the prolongation of kenogen phase and that it occurred to a greater degree in men with androgenetic alopecia. These two changes in hair cycling with age result in loss of both hair density and hair volume. They also reported that it seems likely that the same age-related changes in hair cycling also occur in women.

*Corresponding author: Dalia Gamal Aly, Department of Dermatology and Venereology, National Research Center, El-Bhoos St., Dokky, Giza, Egypt, Tel: +201223720243; E-mail: dalia.g.aly@gmail.com

Received: August 18, 2014; Accepted: September 26, 2014; Published: September 28, 2014

Citation: Aly DG (2014) Hair: A Review of Prominent Scalp Hair Changes throughout a Females' Life. Pigmentary Disorders 1:136. doi:10.4172/JPD.1000137

Copyright: (c) 2014 Aly DG. The terms of the Creative Commons Attribution License, which permits unrestricted use, distribution, and reproduction in any medium, provided the original author and source are credited. 


\section{Hair Parameters}

Evaluation of scalp hair requires the assessment of several variables such as linear growth rate, hair density and hair diameter. Such information provides essential details for determining normal morphology, understanding changes arising from disease, and for developing adequate treatment [7].

\section{Rate of hair growth}

The scalp contains, on average, 100,000 hairs. The rate of hair growth varies from region to region, as well as with sex and age. Hair grows about 6 inches a year for most people. The average rate of growth of hair on the crown of the scalp of a female is approximately $0.5 \mathrm{~mm} / 24 \mathrm{~h}$, being slightly less on the margins, while on the vertex it is $0.45 \mathrm{~mm} / 24 \mathrm{~h}$, and there are no variations diurnally or during the menstrual cycle. Although scalp hair grows faster in women than in men, the rate before puberty is greater in boys than in girls [4].

Normally $10 \%$ to $15 \%$ of these hairs are in the telogen phase. According to the American Academy of Dermatology, shedding of 100 to 150 telogen hairs per day is normal and on the days when hair is washed, people can lose up to 250 strands [3].

\section{Hair density}

Hair density quantifies the number of hairs per unit area. Scalp hair follicles are arranged in groups known as follicular units. At high hair densities, follicular units may contain three to five hairs. At low hair densities, follicular units may comprise only one or two hairs [2].

With growth, there is a progressive reduction in follicle density, which continues until adult life, as skin surface area increases. This occurs to a greater degree over the trunk and limbs than over the head so that the reduction in follicle density is less marked on the head than elsewhere. There is also racial variation in scalp hair density: average scalp hair density in white people varies between 250 and 320 hairs $/ \mathrm{cm}^{2}$ [8], in Africans it's about $187 / \mathrm{cm}^{2}$ [9] and African Americans $171 / \mathrm{cm}^{2}$ which is lower than in white people, and it is even lower in Koreans as it is $128 / \mathrm{cm}^{2}$ [10]. It has been reported that in normal females, the top of the head shows the highest mean hair density, followed by front, back, and sides in decreasing order [11].

Birch et al., [8] measured hair density on the mid/frontal scalp in an unselected sample of women. At all ages there was a wide, normally distributed scatter in hair density. Density declined gradually from the mid-thirties onward from $293 \pm 61.3$ hairs $\mathrm{cm}^{2}$ at age 35 years to $211 \pm 55.1$ hairs $\mathrm{cm}^{2}$ at age 70 years. A clinical assessment of whether subjects showed evidence of hair loss was also made. The majority of subjects classified as having hair loss had below average hair densities but interestingly a few had above average densities and some subjects with below average hair densities were classified as having no hair loss, indicating that factors besides hair density affect the clinical perception of hair loss [11].

\section{Hair diameter}

One of the biggest concerns for the aged female is the decrease in hair volume. The only factor that has been suggested to account for this age dependant decrease is the decrease in hair diameter [12].

The diameter of a human hair does not have a standard value due to several factors as genetic factors and hair color. Black hair is thicker than red hair. Age is also another factor. Babies and young children have finer hair than adults. As a person grows up, their hair becomes thicker and stronger [1]. Kang et al., [11] reported that in normal females, hair on the back of the head had the thickest diameter, followed by the sides, top, and front in decreasing order.

Birch et al., [8] measured hair shaft diameters in hair samples taken from 294 women. Mean hair diameter, increased from age 22 years to a peak in early- to mid-thirties and then gradually declined with advancing age. There was considerable variation in mean diameters between individuals.

\section{Differences in Hair Parameters between Pre- and Postmenopausal Women}

In women without overt hair loss there are significant differences in hair growth characteristics between pre- and postmenopausal women, specifically hair growth rate, density, percent anagen, hair diameters and hair diameter distributions. This is more pronounced in the frontal compared to the occipital scalp. It is known from embryologic and patterning studies that the origin of the frontal and occipital scalp is different and that the follicles from these two areas respond differently to hormonal influences [5].

In a British study, 424 women aged 18-99 years were examined. Hair characteristics were compared on frontal and occipital scalp in pre and post menopausal women. Changes in hair measures were accentuated in frontal scalp compared to the occipital scalp in postmenopausal women [13].

In all of the studied women, hair density was higher on frontal scalp than the occipital scalp. Frontal and occipital hair density showed a wide range of values but showed a decreasing trend after age after 40 indicating that chronologic age but not menopause status dictates alterations in hair density [5].

Hair diameter and hair diameter distribution showed that the mean hair diameter was significantly lower in the postmenopausal women relative to pre-menopausal. It was shown to increase until the age of 40 years, and then decreases after that age. Furthermore, hair diameter distribution showed a change or downshift in the larger diameter hair fiber in pre/postmenopausal while small diameter hairs remained essentially unchanged [14]. This suggests that the larger diameter hairs may be more susceptible to hormonal changes that occur during menopause [13].

\section{Effect of Pregnancy on Hair Parameters}

The best example of a systemic influence on the human hair growth cycle is pregnancy. During pregnancy, there is an increase in the proportion of follicles in anagen leading to an increase in the ratio of anagen/ telogen hairs, although it is not clear whether this is caused by prolongation of anagen or more rapid shedding of telogen hairs. There is also an increase in hair growth rate and diameter. Although higher estrogen levels, likely, play a role in this alteration of hair biology, it is difficult to attribute the phenomenon to estrogen alone because of the complex hormonal changes seen in pregnancy including increases in progesterone and prolactin [15]. Following childbirth, large numbers of follicles enter telogen, leading to increased shedding from about 3 months postpartum (postpartum telogen effluvium) [16].

\section{Extrinsic Factors Leading to Premature Aging of the Hair}

\section{Hair weathering}

Weathering represents the wear and tear that mainly affects the free end of the growing hair fiber. In normal hair, the damage is most prominent only near the tip of scalp hair, which often appears pale and 
lusterless with varying degrees of split ends. The hair fiber is potentially susceptible to friction damage from excessive combing and brushing, particularly when wet and also from excessive heat "blown" or curling irons applied to the hair. Chemical treatment of hair as bleaching, coloring, and straightening, is also a major cause of exaggerated hair weathering. The cuticle becomes softened in the course of these procedures where loss of it leads to longitudinal fissures ultimately resulting in hair fractures at these sites [1].

\section{Role of Ultraviolet Radiation (UVR)}

Progressive thinning of scalp hair with age results in a gradual decline in natural protection of the scalp from UVR. While it has been recognized that at least $50 \%$ of UVR-induced damage to the skin is attributable to the UVR, the effects of UVR on hair have received less attention. Photochemical impairment of the hair includes degradation and loss of hair proteins as well as degradation of hair pigment. UVB radiation is responsible for hair protein loss and UVA radiation is responsible for hair color changes. Moreover, clinical observations and theoretical considerations suggest that UVR may have negative effects on hair. It has been described that acute Telogen Effluvium (TE) from prolonged UVR exposure could occur. Also, the production of porphyrins by Propionibacterium sp. in the pilosebaceous duct, and their photo-activation leading to oxidative tissue injury contributing to follicular micro-inflammation was reported [17].

\section{Physiologic aging of the hair (Hair graying)}

One of the most obvious signs of human ageing is the loss of hair pigmentation, known as graying or canities. While the normal incidence of hair graying is $34 \pm 9.6$ years in Caucasians and $43.9 \pm 10.3$ years in Africans, it has been described that, by 50 years of age, $50 \%$ of people have $50 \%$ gray hair. Women will usually start around the perimeter of the hairline. Gradually, the gray works its way back through the top, sides, and back of the hair. The rate at which an individual turns gray depends on genetics. Hair is said to gray prematurely if it occurs before the age of 20 in Caucasians and before 30 in Africans [1].

The cellular and molecular origins of graying are incompletely understood. The color of hair mainly depends on the presence or absence of melanin pigment. Skin and hair melanins are formed in cytoplasmic organelles called melanosomes, produced by the melanocytes, and are the product of a complex biochemical pathway (melanogenesis) with tyrosinase being the rate limiting enzyme [18].

Gray hair is caused by one or more impairments in melanocytekeratinocyte functions including: reduction in tyrosinase activity in the melanocyte, suboptimal melanocyte-keratinocyte interactions, senile loss of melanocytes and defective migration of melanocyte cells from their reservoir in the outer root sheath to their functional location near the dermal papilla [19]. Additionally, the melanogenic activity of pigmented melanocytes, continuing for up to 10 years in some hair follicles, generates large amounts of reactive oxygen species. If not adequately removed, an accumulation of these reactive oxidative species will generate significant oxidative stress. It is possible that the antioxidant system becomes impaired with age leading to damage to the melanocyte itself from oxidative stress [20].

Several investigators studied the differences between pigmented and gray hair fibers drawn from the same individual and found that pigmented hair was characterized by a larger cross-sectional diameter than gray hair. It was also observed that gray hair fibers were coarser, less manageable and exhibited greater weathering than their pigmented counterparts. Gray hair is said to often fail to hold a temporary or permanent set, and to be more resistant to incorporating artificial color, both of which suggest changes to the underlying substructure of the hair fiber. Also, the growth rate of gray hair was approximately $10 \%$ greater than pigmented hair [19].

\section{Hair Loss in Females}

\section{Female pattern hair loss}

The most common cause of hair loss in females is female-pattern hair loss (FPHL), which is frequently referred to as androgenetic alopecia. However, the role of androgens in this type of hair loss remains uncertain. The condition is often considered familial. It is generally assumed that genetic factors predispose to FPHL but this has yet to be verified [2].

Estimates of its prevalence in women have varied widely, though recent studies claim that $16 \%$ of women aged less than 50 years are affected, increasing to a proportion of $30-40 \%$ of women aged 70 years and over [21]. It is assumed that the genetically predisposed hair follicles are the target for androgen-stimulated hair follicle miniaturization, leading to gradual replacement of large, pigmented hairs (terminal hairs) by barely visible, depigmented hairs (vellus hairs) in affected areas with consequent loss of hair density. The net result is that follicular units that normally have three to five terminal hairs only have one to two terminal hairs. Whether this is a distinct entity or a continuum from normal hair follicle ageing is not known but it clearly affects some women more than others [7].

Female pattern hair loss affects the central portion of the scalp, sparing the frontal hairline, and is characterized by a wider midline part on the crown than on the occipital scalp. In some women, hair thinning over the lateral area of the scalp also occurs. The severity of hair loss is staged according to the Ludwig classifications [22] in which increasing stages (I to III) correspond to increasing widths of the midline part. If hair thinning is more evident in the frontal portion of the scalp, the part may resemble a fir tree in what is known as a "Christmas tree pattern" behind the frontal hair line. Other manifestations include hair thinning on the lateral scalp and male patterns involving thinning on the fronto-temporal and vertex areas of the scalp $[13,23]$.

\section{Is Female Pattern Hair Loss Androgen-Dependent?}

Testosterone is the major circulating androgen; the effect of it on hair growth is mediated by its conversion to the more potent metabolite $5 \alpha$-dihydrotestosterone (DHT) via the action of $5 \alpha$-reductase enzyme. Binding of DHT to the androgen receptor (AR) then occurs. The predisposed scalp exhibits high levels of DHT, and increased expression of the AR [13].

The majority of women with FPHL have normal serum androgen levels [7]. However, other observations suggest that balding is common in women with hyperandrogenism, where it is often associated with other features of androgen excess such as hirsutes and oligo- or amenorrhea [13]. On the other hand, one study showed hyperandrogenemia in $38.5 \%$ of women with moderate-to-severe FPHL; approximately one quarter of these women had no other signs of hyperandrogenemia, such as hirsutism or menstrual disturbances [24].

It is not yet known whether hair loss relates to a high level of sensitivity of scalp hair follicles to normal female levels of androgens or is due to another, non-androgenic mechanism. It would seem that for women, androgens in the normal female range are sufficient to induce early baldness in women with a strong genetic predisposition. In women without a strong predisposition, balding will not occur until 
later in life unless endogenous androgen production is increased or drugs with androgen-like activity are taken [4].

\section{Role of Inflammation in Female Pattern Hair Loss}

The implication of microscopic follicular inflammation in the pathogenesis of FPHL has emerged from several studies. Inflammation might occur near the infundibulum where the microbial colonization of the follicular infundibulum with Propionibacterium sp., Staphylococcus sp., Malassezia sp., or other members of the transient flora, could lead to the generation of an inflammatory response by the microbial toxins. Alternatively, keratinocytes themselves may respond to chemical stress from irritants, pollutants, and UVR, by producing radical oxygen species and nitric oxide, and by releasing intracellularly stored interleukin (IL)-1a. This pro-inflammatory cytokine by itself has been shown to inhibit the growth of isolated hair follicles in culture [1].

\section{Telogen Effluvium}

Another common cause of hair loss in females is TE. This condition results from an abrupt shift of large numbers of anagen hairs to telogen hairs on the scalp, with a corresponding change in the ratio of anagen hair to telogen hair from the normal ratio of 90:10 to 70:30. It is not unusual for women with TE to lose more than 300 hairs per day. This form of hair loss generally begins approximately 3 months after a major illness or other stress (e.g., surgery, rapid weight loss, nutritional deficiency, high fever, or hemorrhage) or hormonal derangement (e.g., thyroid dysfunction) [7].

It has also been reported after the initiation of treatment with certain medications as oral contraceptives, and hormone replacement therapy [25]. Telogen effluvium is distinct from anagen effluvium, the hair loss associated with chemotherapeutic agents that cause immediate destruction and release of anagen hair [16]. If the cause of TE is removed, hair loss lasts for up to 6 months after removal of the trigger. Cosmetically significant re-growth can take 12 to 18 months [7].

Some women complain of a sustained increase in daily hair shedding for more than 6 months, usually accompanied by loss of hair volume, and without an identifiable cause. This presentation has been termed 'Chronic Telogen Effluvium' (CTE). In some patients, this type of hair loss lasts for years [2].

A study from Australia reported that around $60 \%$ of patients with CTE showed histological evidence of follicular miniaturization, suggesting that early FPHL is responsible for causing excessive shedding in the majority of patients with CTE. Increased hair shedding could be the result of age-related shortening of the hair cycle, which is more noticeable in women with naturally high hair density and long hair styles. It has been proposed, but not confirmed, that decreasing estrogen levels may contribute to this clinical phenomenon [26].

Chronic telogen effluvium has also been ascribed to iron deficiency. This idea is however, controversial and there are no published studies showing its reversal by iron supplementation [27].

\section{Pharmacologic Interventions and Care of Hair}

\section{Treatment of female pattern hair loss}

Therapies for FPHL include topical minoxidil, anti-androgen medication, and hair transplantation in selected patients. Six months to 1 year of treatment may be required before there is considerable improvement [7].

Minoxidil solution: Minoxidil is a potent vasodilator that is effective orally for severe hypertension. When applied topically in a lotion or foam vehicle, it increases terminal hair density in up to $30 \%$ of individuals $[4,20]$.

Topical 2\% minoxidil solution is approved by the Food and Drug Administration (FDA) for women with thinning hair due to FPHL. Topical minoxidil has been shown to arrest hair loss or to induce mild to moderate hair re-growth in approximately $60 \%$ of women with FPHL [7]. If treatment is stopped, clinical regression occurs within 6 months, to the state of baldness that would have existed if treatment had not been applied. Patients should be warned that in order to maintain any beneficial effect, applications must continue twice daily for the rest of their lives [4].

The use of $5 \%$ minoxidil may be considered in women who do not have a response to the $2 \%$ formulation or who want more aggressive management. A double-blind, randomized trial comparing a 5\% minoxidil solution with a $2 \%$ minoxidil solution used twice daily in women with mild-to-moderate FPHL showed significantly greater patient satisfaction with the $5 \%$ preparation. Although it is prescribed by many dermatologists in practice, the $5 \%$ minoxidil solution is not FDA-approved for use in women [28].

Anti-androgen therapies: If anti-androgen agents are used in women of reproductive age, an oral contraceptive should be prescribed concomitantly, since these agents are known teratogens [7].

Spironolactone is a synthetic steroid, structurally related to aldosterone, which acts by competitively blocking androgen receptors. It also weakly inhibits androgen biosynthesis. Its primary use is as a diuretic and antihypertensive. [4].

There are no controlled trials in FPHL. However, there is one report that women treated for 1 year with spironolactone showed less hair loss than in an untreated group. Dosage ranges from 100 to 300 $\mathrm{mg} /$ day $[4,27]$.

Cyproterone acetate is an androgen receptor blocker and potent progestin. It also has an anti-gonadotrophic effect. It has been in common usage for over 40 years in the treatment of hirsutism [7].

One study demonstrated that the use of cyproterone acetate (50 to $100 \mathrm{mg}$ daily for 10 days of the menstrual cycle) or spironolactone ( $200 \mathrm{mg}$ daily) in women with FPHL, showed that $80 \%$ of women had either hair re-growth or stabilization of hair loss, but this study was uncontrolled [29].

In a randomized trial comparing topical $2 \%$ minoxidil solution plus an oral contraceptive versus cyproterone acetate (52 mg per day) plus an oral contraceptive in women with FPHL, the latter combination resulted in greater hair density in women with hyperandrogenism, whereas in women without hyperandrogenism, minoxidil had a greater effect [30].

Flutamide is a non-steroidal anti-androgen that acts by inhibiting androgen uptake and by inhibiting nuclear binding of androgen within the target tissue. One study suggested that flutamide is superior to cyproterone acetate and finasteride in the treatment of androgenetic alopecia [31].

Finasteride is a $5 a$-reductase inhibitor. In two uncontrolled studies, finasteride (Propecia) at a minimum dose of $2.5 \mathrm{mg}$ per day appeared to have a benefit for women with FPHL [32]. However, in a controlled trial involving postmenopausal women with FPHL, treatment with it at a dose of $1 \mathrm{mg}$ per day was not significantly better than placebo [33].

Hair transplantation: Hair surgery is increasingly used to treat 
many women with FPHL [34]. One or two sessions are usually sufficient for a cosmetically acceptable result. Many surgeons use minoxidil therapy in patients who have undergone hair transplantation although this strategy has also not been rigorously studied [35].

\section{Treatment of telogen effluvium}

The most important aspect in the management of TE is educating the patient about the normal hair cycle as well as the relationship between triggers and the timing of hair loss [36]. If the trigger is identified and removed, shedding is short-lived and no further treatment is required [3].

In general, adequate nutrition is essential in TE. Any underlying scalp inflammation as seborrheic dermatitis or psoriasis should be treated with an anti-dandruff shampoo and a topical corticosteroid. Thyroid disease, systemic illnesses, and infections should also be treated [36]

For acute telogen hair loss, and chronic TE, biotin and zinc replacement can support hair re-growth. Applying topical minoxidil (Rogaine) $2 \%$ and $5 \%$ to the scalp once a day can be useful [3].

\section{Possibilities for reversal of hair graying}

Temporary hair darkening has been reported after the ingestion of large doses of $p$-aminobenzoic acid. One study gave $100 \mathrm{mg}$ three times daily to 460 gray-haired individuals. Darkening was obvious in $82 \%$ within $2-4$ months of starting treatment. The hairs turned gray again 2-4 weeks after stopping therapy. The mechanism of action has remained unclear. Side effects were frequent, primarily gastrointestinal upset [1].

In the absence of a natural way to reverse hair graying, hair colorants are the mainstay of recovering lost hair color. The major types of hair colors currently used are: natural coloring (henna), temporary, semipermanent and permanent dyes [37].

Henna, obtained from the plant Lawsonia alba, is a naturally occurring hair colorant. Although the color can add red highlights to hair, occasionally it may come out looking orange on gray hair [1].

Temporary hair colorants consist of large complex organic structures that do not penetrate the cuticle. The colors are not intense but are capable of covering gray hair in a subtle way. The colorant washes out with the next shampoo. Typical product forms include shampoos and sprays. Semi-permanent colorants consist of small molecules that penetrate the cuticle. These compounds color gray hair very nicely, are easily applied in a lotion or foam at home, and last for six to ten shampoos [4]. The most frequently used hair colorant is permanent hair dye. In permanent hair coloring, the formation of colored molecules from their precursors occurs inside the hair fibers as a result of oxidation by hydrogen peroxide. The advantage of permanent color is that the color withstands normal hair washing [1].

\section{Photo-protection of hair and scalp}

Although hats provide the best protection of the scalp from UVR, not all patients find them convenient. Hair dyes may also protect the hair against photo damage. Cinnamidpropyltrimonium chloride, an UV absorber, delivered from a shampoo system, was also found suitable for photo protection of hair [38]. Additionally, solid lipid nano particles which are novel carriers of UV blockers for the use on skin and hair, offer photo protection on their own by reflecting and scattering UVR [39].
Systemic photo protection has been the focus of more recent investigation. Preclinical studies showed the photo protective properties of supplemented antioxidants, as beta-carotene (provitamin A), vitamin E, and vitamin C. Nevertheless, combining these compounds with known sunscreens is needed to maximize photo-protection [1].

\section{Anti-aging compounds}

Advances in the care of aging hair and scalp are "anti-aging" compounds. Due to water dilution and short contact time, antiaging compounds do not have any effect in shampoos. Antioxidants in shampoos, such as vitamin $\mathrm{C}$ and $\mathrm{E}$, protect fatty substances in the shampoo from oxidation, and not the scalp. Topical anti-aging compounds of current interest are green tea polyphenols, selenium, copper, melatonin, and as yet unidentified substances from traditional Chinese medicine. Hormonal anti-aging protocols containing recombinant human growth hormone report improvement of hair thickness and structure in $38 \%$ of patients, in some cases darkening of hair, and in few increased hair growth [40].

\section{Future Directions}

There is an increasing interest in the hair follicular route for delivery of active compounds affecting the hair. Current research activities focus on topical liposome targeting for melanins, genes, and proteins selectively to hair follicles for therapeutic and cosmetic modification of hair. For example, topical liposome selective delivery to hair follicles has demonstrated the ability to color hair with melanin. Finally, yet another line of research in the quest of new treatments for hair loss is tissue engineering with cells of hair follicular origin with inductive properties [1].

\section{Conclusion}

Within the female population, there is a gradual decline in production of scalp hair with advancing age although there is a wide variation in timing and degree. This process appears to start from the mid-thirties and encompasses alterations in hair cycling (reduction in anagen duration and prolongation of telogen), and a decline in both hair density and hair diameter. These changes are exaggerated in FPHL in which loss of hair density is also the result of follicular miniaturization as well as changes in the hair cycle. Hair Graying is one of the most obvious signs of hair ageing and nowadays, there is also emerging evidence that environmental factors as UVR play a role in this process.

\section{References}

1. Trüeb RM (2006) Pharmacologic interventions in aging hair. Clin Interv Aging 1: 121-129.

2. Messenger AG (2011) Hair through the female life cycle. $\mathrm{Br} \mathrm{J}$ Dermatol 165 Suppl 3: 2-6

3. Harrison S, Bergfeld W (2009) Diffuse hair loss: its triggers and management Cleve Clin J Med 76: 361-367.

4. Messenger AG, De Berker DAR, Sinclair RD (2010) Disorders of Hair, In: Rook's Textbook of Dermatology. (8thedtn). Wiley-Blackwell, Oxford, UK.

5. Tajima M, Hamada C, Arai T, Miyazawa M, Shibata R, et al. (2007) Characteristic features of Japanese women's hair with aging and with progressing hair loss. J Dermatol Sci 45: 93-103.

6. Courtois M, Loussouarn G, Hourseau C, Grollier JF (1995) Ageing and hair cycles. Br J Dermatol 132: 86-93.

7. Shapiro J (2007) Clinical practice. Hair loss in women. N Engl J Med 357 1620-1630.

8. Birch MP, Messenger JF, Messenger AG (2001) Hair density, hair diamete and the prevalence of female pattern hair loss. Br J Dermatol 144: 297-304. 
Citation: Aly DG (2014) Hair: A Review of Prominent Scalp Hair Changes throughout a Females' Life. Pigmentary Disorders 1:136. doi:10.4172/ JPD.1000137

Page 6 of 6

9. Loussouarn G (2001) African hair growth parameters. Br J Dermatol 145: 294 297.

10. Lee HJ, Ha SJ, Lee JH, Kim JW, Kim HO, et al. (2002) Hair counts from scalp biopsy specimens in Asians. J Am Acad Dermatol 46: 218-221.

11. Kang H, Kang TW, Lee SD, Park YM, Kim HO, et al. (2009) The changing patterns of hair density and thickness in South Korean women with hair loss: clinical office-based phototrichogram analysis. Int J Dermatol 48: 14-21.

12. Mamada A, Nakamura K (2007) A study of the volume and bounce decrease in hair with aging using bending elasticity measurements. J Cosmet Sci 58 485-494.

13. Birch MP, Lalla SC, Messenger AG (2002) Female pattern hair loss. Clin Exp Dermatol 27: 383-388.

14. Mirmirani P, Luo F, Youngquist SR, Fisher BK, Li J, et al. (2010) Hair Growth parameters in pre- and postmenopausal women, In: Aging Hair. (1stedtn) Springer, Heidelberg, Berlin.

15. Nissimov J, Elchalal U (2003) Scalp hair diameter increases during pregnancy. Clin Exp Dermatol 28: 525-530.

16. Muallem MM, Rubeiz NG (2006) Physiological and biological skin changes in pregnancy. Clin Dermatol 24: 80-83.

17. Trüeb RM (2003) Is androgenetic alopecia a photoaggravated dermatosis? Dermatology 207: 343-348.

18. Tobin DJ, Paus R (2001) Graying: gerontobiology of the hair follicle pigmentary unit. Exp Gerontol 36: 29-54.

19. Kaplan PD, Polefka T, Grove G, Daly S, Jumbelic L, et al. (2011) Grey hair: clinical investigation into changes in hair fibres with loss of pigmentation in a photoprotected population. Int J Cosmet Sci 33: 171-182

20. Van Neste D, Tobin DJ (2004) Hair cycle and hair pigmentation: dynamic interactions and changes associated with aging. Micron 35: 193-200.

21. Wang TL, Zhou C, Shen YW, Wang XY, Ding XL, et al. (2010) Prevalence of androgenetic alopecia in China: a community-based study in six cities. $\mathrm{Br} J$ Dermatol 162: 843-847

22. Ludwig E (1977) Androgenetic alopecia. Arch Dermatol 113: 109

23. Olsen EA (2001) Female pattern hair loss. J Am Acad Dermatol 45: S70-80.

24. Orme S, Cullen DR, Messenger AG (1999) Diffuse female hair loss: are androgens necessary? Br J Dermatol 141: 521-523.

25. Bergfeld WF (2008) Telogen effluvium. In: Hair and Scalp Diseases: Medical Surgical, and Cosmetic Treatments. Informa Health Care, London, UK.
26. Sinclair R, Jolley D, Mallari R, Magee J (2004) The reliability of horizontally sectioned scalp biopsies in the diagnosis of chronic diffuse telogen hair loss in women. J Am Acad Dermatol 51: 189-199.

27. Rushton DH, Bergfeld WF, Gilkes JJ, Van Neste D (2011) Iron deficiency and hair loss--nothing new? J Am Acad Dermatol 65: 203-204.

28. Lucky AW, Piacquadio DJ, Ditre CM, Dunlap F, Kantor I, et al. (2004) A randomized, placebo-controlled trial of $5 \%$ and $2 \%$ topical minoxidil solutions in the treatment of female pattern hair loss. J Am Acad Dermatol 50: 541-553.

29. Sinclair R, Wewerinke M, Jolley D (2005) Treatment of female pattern hair loss with oral antiandrogens. Br J Dermatol 152: 466-473.

30. Vexiau P, Chaspoux C, Boudou P, Fiet J, Jouanique C, et al. (2002) Effects of minoxidil $2 \%$ vs. cyproterone acetate treatment on female androgenetic alopecia: a controlled, 12-month randomized trial. Br J Dermatol 146: 992-999.

31. Carmina E, Lobo RA (2003) Treatment of hyperandrogenic alopecia in women Fertil Steril 79: 91-95

32. Iorizzo M, Vincenzi C, Voudouris S, Piraccini BM, Tosti A (2006) Finasteride treatment of female pattern hair loss. Arch Dermatol 142: 298-302.

33. Price VH, Roberts JL, Hordinsky M, Olsen EA, Savin R, et al. (2000) Lack of efficacy of finasteride in postmenopausal women with androgenetic alopecia. J Am Acad Dermatol 43: 768-776.

34. Unger WP, Unger RH (2003) Hair transplanting: an important but often forgotten treatment for female pattern hair loss. J Am Acad Dermatol 49: 853-860.

35. Avram MR, Cole JP, Gandelman M, Haber R, Knudsen R, et al. (2002) The potential role of minoxidil in the hair transplantation setting. Dermatol Surg 28 : 894-900.

36. Bergfeld WF, Mulinari-Brenner F (2001) Shedding: how to manage a common cause of hair loss. Cleve Clin J Med 68: 256-261.

37. Neuser F, Schlatter H (2010) Hair dyes in Cosmetic Dermatology: Products and Procedures. Blackwell Publishing Ltd., USA.

38. Gao T, Bedell A (2001) Ultraviolet damage on natural gray hair and its photoprotection. J Cosmet Sci 52: 103-118.

39. Wissing SA, Müller RH (2001) Solid lipid nanoparticles (SLN)--a novel carrier for UV blockers. Pharmazie 56: 783-786.

40. Lurie R, Ben-Amitai D, Laron Z (2004) Laron syndrome (primary growth hormone insensitivity): a unique model to explore the effect of insulin-like growth factor 1 deficiency on human hair. Dermatology 208: 314-318. 